\title{
MONITORIZAÇÃO ÓPTICA DO DESEMPENHO EM SISTEMAS DE COMUNICAÇÕES BASEADA EM HISTOGRAMAS DE AMPLITUDE ASSINCRONAMENTE AMOSTRADOS
}

\author{
Paulo S. André, A. L. J. Teixeira, M. J. N. Lima, F. da Rocha, J. L. Pinto, T. Almeida e M. Pousa
}

\begin{abstract}
Resumo - A estimativa do desempenho dos sistemas de comunicações ópticas é um aspecto fundamental no desenvolvimento das futuras redes completamente ópticas. No âmbito deste trabalho pretendeu-se desenvolver técnicas que permitam estimar o desempenho de um sinal óptico multiplexado no comprimento de onda, distorcido pela dispersão cromática, ruído de emissão espontânea dos amplificadores e efeitos não lineares, utilizando histogramas de amplitude assincronamente amostrados. Foi verificado experimentalmente um modelo numérico desenvolvido para estimar o factor $Q$ a partir de histogramas assíncronos em função da relação sinal / ruído, tempo de subida e formato dos impulsos. Também propomos um modelo para aceder directamente a BER de um sinal DWDM a partir de histogramas assincronamente amostrados. Os resultados experimentais obtidos indicam que este método pode monitorizar a degradação do sinal óptico, confirmando a aplicabilidade desta técnica para monitorização óptica do desempenho para redes fotónicas transparentes.
\end{abstract}

Palavras-chave: Multiplexagem no comprimento de onda, monitorização óptica do desempenho, amostragem assíncrona, proteção de redes, qualidade de serviço.

\begin{abstract}
The performance monitoring in optical networks is a crucial aspect in the deployment of the future transparent photonic networks. In this paper we evaluate the asynchronous $Q$ factor from a dense wavelength division multiplexed optical signal distorted by chromatic dispersion, amplified spontaneous emission noise and non-linear effects, using amplitude histograms. It was experimentally confirmed the asynchronous histogram numerical model developed to estimate the $Q$ factor as function of the signal to noise ratio, rise time and format of the optical pulses. We also propose a model to access directly the bit error rate of a DWDM optical signal, from the asynchronously detected amplitude histograms. The obtained experimental results indicate that this method can monitor the signal performance degradation, confirming the applicability of this technique as optical performance monitor for transparent photonics networks.
\end{abstract}

Paulo André e João Lemos Pinto estão no Instituto de Telecomunicações e no Departamento de Física da Universidade de Aveiro. Antônio Teixeira, Mário Lima e José Ferreira da Rocha estão no Departamento de Eletrônica e Telecomunicações da Universidade de Aveiro. Teresa Almeida e Marcelino Pousa estão na Portugal' Telecom Inovação. Todos os autores são investigadores no Instituto de Telecomunicações, Campus de Santiago, Aveiro, Portugal. E-mails: pandre@av.it.pt, teixeira@ua.pt, mmcc@ua.pt, frocha@ieee.org. jlp@fis.ua.pt, teresa@ ptinovacao.pt, mpousa@ptinovacao.pt. Editor de Área responsável: Renato Baldini Filho. Artigo submetido 03/Jul/2002, revisado em 05/Nov/2002, aceito em 01/Abr/2003.
Keywords: Wavelength division multiplexing, optical performance monitoring, asynchronous sampling, network protection, quality of service.

\section{INTRODUÇÃO}

A técnica de multiplexagem no comprimento de onda veio permitir a expansão da capacidade dos sistemas de comunicações ópticas ponto a ponto existentes, aproveitando o aumento da capacidade de transporte providenciada pela enorme largura de banda das fibras instaladas. $\mathrm{O}$ próximo passo evolutivo será a migração para redes fotónicas completamente ópticas e transparentes que ofereçam esquemas de encaminhamento no domínio óptico, substituindo os actuais esquemas baseados em circuitos electrônicos. Tal, implica que em qualquer nó de rede possam coexistir sinais com diversos ritmos de transmissão, vários formatos de modulação e diversos protocolos digitais. Por outro lado, a gestão, controlo e sobrevivência num cenário de multi-vendedores, multi-operadores e multi-consumidores requer a habilidade de medir o desempenho do canal óptico, detectar degradações dos sinais e providenciar meios de localização de falhas, bem como o seu isolamento. Desta forma, assegura-se a manutenção constante da qualidade de serviço (QoS) oferecida.

Esta evolução dos sistemas ópticos ponto a ponto para redes ópticas multi-comprimento de onda transparentes, torna difícil a monitorização da qualidade de serviço dos canais, sem que se faça a análise directa da informação transmitida, ou seja, sem recorrer a métodos intrusos. A substituição dos nós elétricos da rede, por nós de rede a operarem no domínio óptico, cria também a necessidade de dotar as redes ópticas com módulos capazes de avaliarem a qualidade do sinal na camada óptica de uma forma econômica.

Com o objectivo de monitorizar o sinal em redes ópticas sem o conhecimento dos serviços suportados pelo cliente, propõe-se neste trabalho, a implementação de um módulo de baixo custo, capaz de monitorizar o sinal óptico, com base numa amostra retirada do sistema de transmissão do cliente. Na secção 2 descrevem-se os métodos de análise de desempenho dos sistemas digitais de comunicações ópticas, nomeadamente, a taxa de erro do bit e do factor $Q$. Na secção 3 apresenta-se um resumo dos métodos utilizados para a realização da monitorização óptica do desempenho em sistemas de transmissão. Na secção 4 faz-se um estudo da utilização de histogramas assíncronos para a monitorização de redes ópticas. É apresentado um modelo teórico para histogramas assincronamente amostrados e um modelo que permite estimar a taxa de erro do bit com base em histogramas de amplitude assincronamente amostrados de um sinal óptico degradado pelos efeitos lineares e não lineares 
da fibra óptica, bem como o ruído introduzido pelos amplificadores ópticos.

\section{AVALIAÇÃO DO DESEMPENHO NOS SISTEMAS DIGITAIS}

O critério utilizado para analisar o desempenho de sistemas digitais é a taxa de erro do bit, BER, definida como a probabilidade média de identificação incorrecta de um bit pelo circuito de decisão. O sinal eléctrico recebido pelo circuito de decisão é amostrado num determinado instante de decisão. Os valores amostrados flutuam em torno de valores médios, $\mu_{l}$ e $\mu_{0}$, correspondendo respectivamente aos símbolos lógicos " 1 " e " 0 ". A probabilidade média de erro num sistema binário é dada por:

$$
B E R=p_{0} \cdot P(1 \mid 0)+p_{1} \cdot P(0 \mid 1)
$$

em que $p_{0}$ e $p_{\ell}$ são respectivamente as probabilidades iniciais dos símbolos lógicos "0" e "I". $P(O \mid I)$ e $P(I \mid O)$ são respectivamente as probabilidades de decidir erroneamente um símbolo lógico " 0 " quando é enviado um símbolo lógico "l" e a probabilidade de decidir erroneamente um símbolo lógico "I" quando é enviado um símbolo lógico " 0 ". Assumindo que os símbolos lógicos são equiprováveis e que o nível de decisão é $I_{d}$, pode-se calcular a BER através da expressão:

$$
B E R=\frac{1}{2} \cdot \int_{-\infty}^{I_{l}} p d f_{1}(I) d I+\frac{1}{2} \cdot \int_{I_{d}}^{+\infty} p d f_{0}(I) d I
$$

em que $p d f_{I}(I)$ e $p d f_{0}(I)$ são as funções densidade de probabilidade, associadas respectivamente à recepção do símbolo lógico "1" e "0". A forma funcional das funções $p d f_{\text {I.o }}$ depende da estatística das fontes de degradação responsáveis pelas flutuações do sinal. Considerando uma aproximação gaussiana para a estatística do sinal analisado, pode-se determinar a probabilidade média de erro [1]:

$$
\begin{aligned}
P(0 \mid 1)= & \frac{1}{\sigma_{1} \cdot \sqrt{2 \pi}} \cdot \int_{-\infty}^{I_{D}} \exp \left(-\frac{\left(I-\mu_{1}\right)^{2}}{2 \cdot \sigma_{1}{ }^{2}}\right) d I= \\
& \frac{1}{2} \cdot \operatorname{erfc}\left(\frac{\mu_{1}-I_{D}}{\sqrt{2} \cdot \sigma_{1}}\right) \\
P(1 \mid 0)= & \frac{1}{\sigma_{0} \cdot \sqrt{2 \pi}} \cdot \int_{I_{D}}^{\infty} \exp \left(-\frac{\left(I-\mu_{0}\right)^{2}}{2 \cdot \sigma_{0}{ }^{2}}\right) d I= \\
& \frac{1}{2} \cdot \operatorname{erfc}\left(\frac{I_{D}-\mu_{0}}{\sqrt{2} \cdot \sigma_{0}}\right)
\end{aligned}
$$

onde $\sigma_{l}^{2}$ e $\sigma_{0}^{2}$ são as variâncias correspondentes a cada um dos símbolos lógicos.

O nível de decisão deve ser escolhido de forma a minimizar a BER do sistema, este mínimo, considerando uma aproximação gaussiana, ocorre para um nível de decisão dado por:

$$
I_{D}=\frac{\sigma_{0} \cdot \mu_{1}+\sigma_{1} \cdot \mu_{0}}{\sigma_{0}+\sigma_{1}}
$$

Nestas condiçôes, pode-se determinar o factor de qualidade $Q$ do sinal, através da expressão:

$$
Q=\frac{\left|\mu_{1}-\mu_{0}\right|}{\sigma_{1}+\sigma_{0}}
$$

Admitindo que a chegada dos símbolos lógicos ao circuito de decisão do receptor têm uma distribuição, gaussiana em torno dos valores médios, a BER relaciona-se com o factor de qualidade do sinal para o nível de decisão óptimo, pela seguinte expressão [1]:

$$
B E R=\frac{1}{2} \cdot \operatorname{erfc}\left(\frac{Q}{\sqrt{2}}\right) \approx \frac{1}{\sqrt{2 \pi} \cdot Q} \cdot \exp \left(-\frac{Q^{2}}{2}\right)
$$

A aproximação feita na expressão anterior é válida para valores de $Q$ superiores a 3, pelo que pode ser utilizada no contexto deste trabalho.

\section{MONITORIZAÇÃO ÓPTICA}

As redes ópticas actuais são geridas, protegidas $\mathrm{e}$ monitorizadas no domínio eléctrico, o que requer a terminação e descodificação dos canais a analisar. Um método de monitorização é a deteç̧ão dos bits de paridade relacionados com a verificação da informação transportada. Como por exemplo, pode-se referir o mecanismo de BIP-8 do protocolo SDH ou do protocolo SONET, que permite uma análise da existência de erros e a subsequente utilização das capacidades de gestão destes protocolos de transmissão para providenciar protecção. Este método é dispendioso e não identifica os bits erróneos, sendo a sua utilização impraticável ao longo do percurso de transmissão, pois implica uma perda de transparência como resultado da enorme variedade de protocolos digitais e de ritmos de transmissão presentes ao longo do percurso [2]. Estes protocolos incluem, entre outros SDH / SONET, PDH, Gigabit Ethernet, ATM e IP.

A habilidade de monitorizar a qualidade do sinal independentemente do formato e do ritmo de modulação é essencial. Consequentemente, a realização das funções de encaminhamento e gestão directamente no domínio óptico, requer o uso de técnicas de monitorização, também, no domínio óptico, sem a necessidade de descodificar a informação contida no protocolo de transmissão digital. A monitorização óptica do desempenho ou OPM (optical performance monitoring) é uma aproximação que permite determinar a qualidade da informação transportada pelo canal e detectar degradações, sem informação da sua origem, história de transporte, formato ou conteúdo. Mais ainda, a OPM deve ser não intrusiva, precisa, não ambígua e barata.

Um sinal óptico que se propague numa rede fotónica transparente encontra-se sujeito a diversas perturbações: $i$ ) ruído provocado por flutuações aleatórias que podem ser tratadas como um processo gaussiano, tal como o ruído de emissão espontânea dos amplificadores ópticos, ou ruído de intensidade relativa de lasers; ii) distorção dos impulsos provocada por não linearidades, dispersão cromática ou dispersão devido aos modos de polarização e iii) diafonia homodina ou heterodina, provocada pela interferência do sinal óptico com outros sinais, com o mesmo comprimento de onda (homodina) ou com comprimentos de onda diferentes (heterodina). Dependendo do número de termos 
interferentes a diafonia pode ser considerada como ruído caracterizado por uma estatística gaussiana $[3,4]$.

Apesar de a BER ser o principal parâmetro para a análise da qualidade de um canal digital, esta pode ser complementada no diagnóstico de problemas por outros parâmetros. Exemplos desses parâmetros complementares são: a relaçāo sinal / ruído óptica (OSNR), factor $Q$, razão de extinção do sinal, potência óptica dos canais, dispersão cromática e PMD. equalização dos canais, diafonia e estabilidade dos comprimentos de onda dos canais.

Várias técnicas de monitorização óptica do desempenho têm sido propostas, nomeadamente, utilizando a OSNR, o factor $Q$ e os histogramas de amplitude. Como a OSNR é calculada a partir de uma medida $d c$, não contabiliza a distorção da forma de onda dos impulsos, induzida pelos efeitos da dispersão cromática e efeitos não lineares. De salientar que mesmo que os efeitos dà distorção não sejam significativos, o valor da OSNR pode variar para um determinado nível da BER. devido à contribuição do ruído de emissão espontânea proveniente de fora da banda espectral ocupada pelo sinal [5].

$O$ factor $Q$ pode ser derivado directamente a partir da BER (expressão (7)), desde que se assuma que a degradação do sinal é causada exclusivamente por ruído gaussiano, podendo assim ser utilizado para OPM [6]. Como foi referido anteriormente, a correspondencia entre a BER e o factor $Q$ requer que a estatística da variável de decisão seja do tipo gaussiano. Esta aproximação não é geralmente satisfeita mesmo para condições simples de propagação. Como o fotodíodo no receptor tem uma função de transferência quadrática, a função densidade de probabilidade à saída do fotodíodo não é gaussiana, mesmo quando o ruído óptico à entrada do receptor apresenta uma distribuição gaussiana. No entanto, durante o processo de filtragem a função densidade de probalidade tenderá para uma função gaussiana, de acordo com o teorema do limite central. Isto ocorre quando o filtro óptico que antecede o receptor tem uma largura de banda $B_{0}$, muito superior ao ritmo de transmissão $R$, usualmente para graus de liberdade $m=B_{o} /(2 R)$ maiores do que 10 [7]. Resumindo, o factor $Q$ pode ser utilizado para avaliar os constrangimentos de propagação causados por ruído, efeitos não lineares, dispersão cromática e dispersão devida aos modos de polarização (PMD) [8].

A avaliação do sinal, independentemente do ritmo de transmissão, sem que seja necessário aceder à informação transmitida pode ser realizada através de histogramas assíncronos [9-11].

A função de monitorização óptica do desempenho de sistemas de comunicações ópticas pode ser dividida em três classes [12]: $i$ ) contínua ou $d c$; ii) baixa velocidade, usualmente inferior a $10 \mathrm{MHz}$ e iii) alta velocidade. Os métodos em contínuo consistem, usualmente, na monitorização da potência óptica média dos canais. Neste caso, por vezes, são aplicados filtros ópticos para a medição selectiva da potência óptica, da OSNR ou da estabilidade dos comprimentos de onda dos canais. Os métodos de baixa velocidade operam através da adição de um sinal analógico ao sinal óptico a analisar. Os métodos de alta velocidade podem ser divididos em duas categorias: análise dos bits de paridade pertencentes aos protocolos digitais de transmissão e histogramas de amplitude.

\section{HISTOGRAMAS DE AMPLITUDE AS- SINCRONOS}

Os histogramas de amplitude assíncronos podem ser obtidos a partir do sinal óptico a analisar, utilizando um acoplador de potência para remover uma amostra do canal. Esta amostra será filtrada por um filtro discriminador que elimina os restantes canais presentes. Seguidamente, o sinal é injectado num fotodíodo, onde é convertido para o domínio eléctrico, e então, amostrado a um determinado ritmo, menor do que o ritmo de transmissão.

Devido à ausência de sincronismo entre o sinal que controla a amostragem e o sinal a amostrar. podem-se obter amostras com qualquer amplitude e em qualquer instante. Logo, não se observa nenhum diagrama de olho aberto que permita escolher a amplitude e o instante de decisão correctos.

Na figura 1 a) mostra-se um diagrama de olho síncrono obtido num osciloscópio digital com uma largura de banda de $40 \mathrm{GHz}$. O sinal tem um ritmo de transmissão de $2.5 \mathrm{Gbit} / \mathrm{s}$, sendo utilizado como sinal de disparo do osciloscópio um sinal síncrono com o sinal a analisar e com uma frequência de $78.125 \mathrm{MHz}$ (ou seja, 1/32 da frequência do sinal de relogio). Na figura 1 b) mostra-se um diagrama de olho assíncrono correspondente ao caso em que é utilizado um ritmo de amostragem de $500 \mathrm{KHz}$. Assim, o sinal não foi sincronizado com um sinal de disparo e o diagrama de olho obtido caracteriza-se por uma distribuição aleatória de amostras ao longo de todo o écran do osciloscópio. Como se observa na figura 1 b) os níveis correspondentes aos símbolos lógicos "1" e " 0 " podem ser identificados por uma presença mais intensa de amostras. $O$ resto do diagrama tem uma distribuição de amostras praticamente uniforme, devido às transições entre os níveis lógicos. Colectando todas as amostras que caem dentro de uma determinada janela temporal é possível obter o respectivo histograma de amplitude. $\mathrm{Na}$ figura 1 b) encontram-sc delimitados os limites da janela de integração utilizada, bem como o histograma de amplitude obtido.

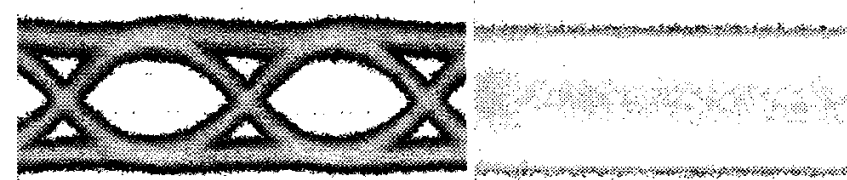

a)

b)

Figura 1. Diagrama de olho, a) síncrono e b) assíncrono. $\mathrm{Na}$ figura b) é visível a janela de integração e o histograma assíncrono correspondente.

\subsection{FACTOR Q ASSÍNCRONO}

Nesta subsecção, pretende-se comparar o factor $Q$ obtido num diagrama de olho síncrono com o factor $Q$ obtido a partir de um diagrama de olho assíncrono. Este estudo foi realizado para dois tipos diferentes de formato dos impulsos 
analisados. Para tal, os sinais eléctricos com sequências de bits pseudo aleatórias (PRBS) de comprimento $2^{7}-1$ e com um ritmo de $2.5 \mathrm{Gbit} / \mathrm{s}$ são injectados em dois tipos distintos de filtros: i) coseno elevado e ii) passa baixo.

$O$ sinal à saída dos filtros é utilizado para polarizar electricamente um modulador Mach-Zehnder (MZ) que codifica o sinal óptico CW proveniente de um laser DFB. O sinal óptico é então injectado na cabeça óptica de um osciloscópio digital de $40 \mathrm{GHz}$. Este osciloscópio permite determinar do factor $Q$ de um diagrama de olho síncrono, $Q_{\text {Sim, }}$ utilizando uma janela de integração variável (neste caso $40 \mathrm{ps}$ ).

Na figura 2 encontra-se a razão entre o factor $Q$ síncrono medido no osciloscópio e o factor $Q$ assíncrono obtido a partir do ajuste a funções gaussianas em torno dos níveis dos símbolos lógicos "l" e "0". Utilizou-se a expressão (6) para calcular o factor $Q$ assíncrono $Q_{\text {Asin }}$, em função da abertura normalizada do diagrama de olho e para os dois formatos dos impulsos atrás descritos. $O$ valor de $Q_{\sin }$ foi mantido constante e igual a 14. Observa-se que, apenas, para uma abertura de olho de 0.66 , a razão entre o factor $Q$ síncrono e assíncrono dos impulsos com formato de coseno elevado coincide com a razão dos factor $Q$ síncrono e assíncrono dos impulsos com formato passa baixo, sendo que as razões tomam um valor unitário.

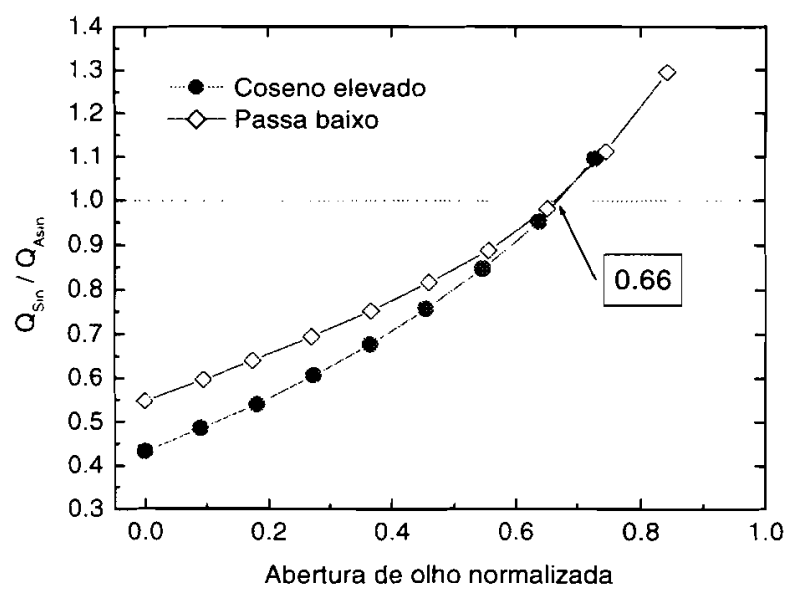

Figura 2. Razão entre o factor $Q$ síncrono e assíncrono em função da abertura de olho considera para a medição do factor $Q$ assíncrono. As linhas que unem os pontos são guias visuais.

Em seguida, mantendo a abertura de olho de 0,66 variouse o factor $Q$ do sinal. Na tabela 1 encontram-se alguns diagramas de olho síncronos e assíncronos para diversos valores do factor $Q$. Na figura 3 mostra-se a comparação para os impulsos com formato do tipo coseno elevado entre o factor $Q$ síncrono e assíncrono. Também se mostra a recta de ajuste linear dos dados.

Os dados das regressões lineares são respectivamente os seguintes: declive de 0.645 , ordenada na origem de $2.11 \mathrm{e}$ coeficiente de correlação de 0.9999. Apesar de se verificar uma relação linear entre o factor $Q$ assíncrono e síncrono, esta relação não tem declive unitário, o que indica um 266 comportamento linear diferente do observado quando $Q_{\text {Sin }}$ tem uma valor em torno de 14.

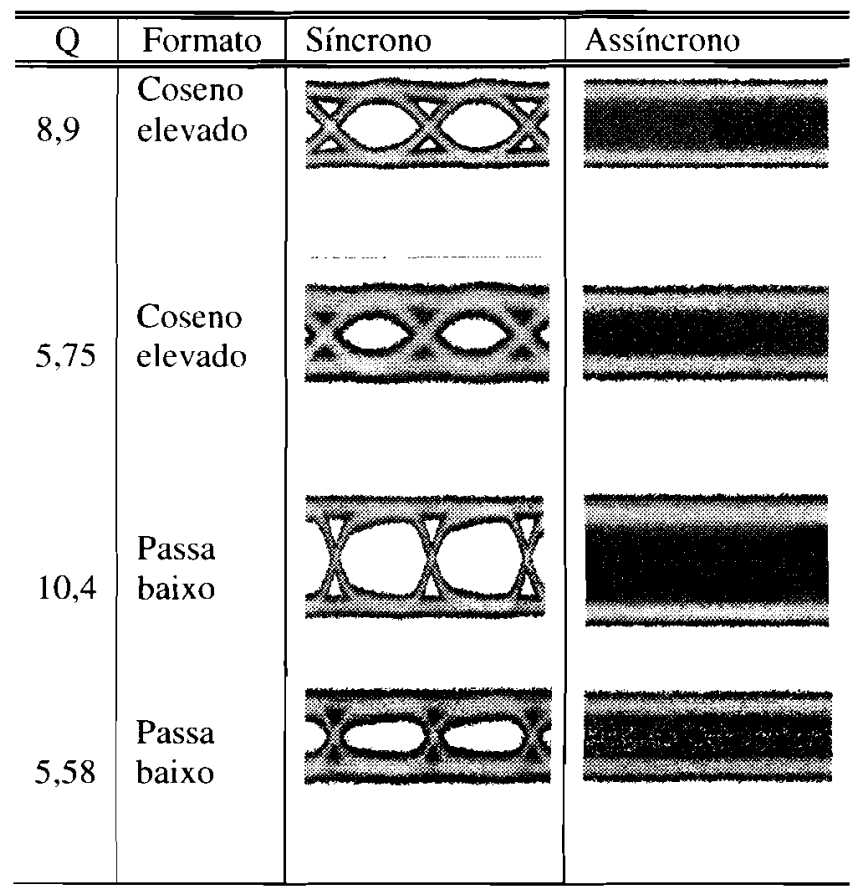

Tabela 1. Diagramas de olho ilustrativos

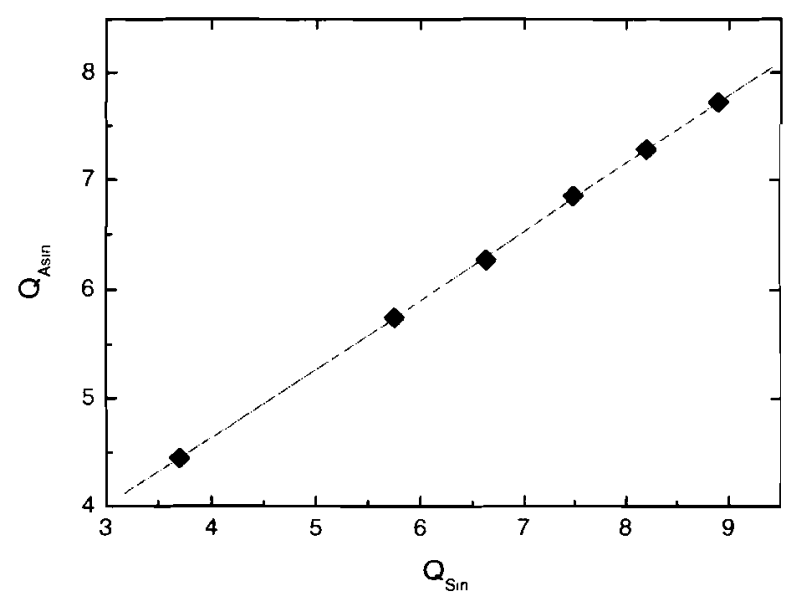

Figura 3. Relação entre o factor $Q$ assíncrono e factor $Q$ síncrono para os impulsos tipo coseno elevado. Os pontos são dados experimentais e a recta o respectivo ajuste linear.

\subsection{MODELO PARA HISTOGRAMAS ASSÍN- CRONOS}

A forma do histograma assíncrono é dependente do formato dos impulsos. No entanto, observa-se que não é possível inferir com precisão os valores das amplitudes médias e desvios padrão associados aos dois níveis lógicos, devido à presença de valores intermédios. A amplitude do vale que existe entre os dois máximos do histograma é dependente do tempo de subida e descida dos impulsos. Estas características permitem extrair os principais parâmetros dos impulsos, como será demonstrado em seguida [13]. 
Por forma a definir um modelo para os histogramas assínronos consideraram-se três formatos para a forma dos impulsos, que se encontram esquematizados na figura $4: i$ ) transição linear ideal, ii) transição coseno elevado e iii) transição passa baixo.

Considerando o esquema superior da figura 4. o tempo $t_{t}$, que os impulsos levam para transitar entre $A_{0}$ e $A_{l}$, ou viceversa é governado pelo parâmetro $\tau$. Estas transições ocorrem segundo um ritmo de repetição periódico de $T$, onde $A_{0}$ e $A$, são respectivamente os níveis associados aos símbolos lógicos " 0 " e "l". O sinal é definido pela variação temporal da sua amplitude e a gama de amplitudes ocupada pelo sinal é dividida em pequenos intervalos, $\Delta$. Assim sendo, a probabilidade de uma componente do sinal permanecer nesses intervalos de amplitude é proporcional ao tempo em que o sinal do impulso se encontra dentro dos limites do intervalo de amplitude.

$\mathrm{Na}$ ausência de ruído e considerando um tempo de aquísição de dados elevado, bem com uma sequência equiprovavél e de comprimento, também, elevado, a probabilidade de se obter cada uma das transições " 1 " $\rightarrow$ "]", "0" $\rightarrow$ "0", "l" $\rightarrow$ " 0 " ou " $0 " \rightarrow$ " 1 " é igual a 1/4. A probabilidade de o sinal se encontrar nos níveis $A_{0}$ e $A_{l}$, ou seja, nos intervalos $\left[A_{0}, A_{O}+\Delta\left[\mathrm{e}\left[A_{l}-\Delta, A_{l}\right]\right.\right.$ é dada por:

$$
P_{0,1}(a)=\frac{1}{4}+\frac{2}{4 \cdot T} \cdot\left(f^{-1}(a)-f^{-1}(a-\Delta)\right)
$$

para $a=A_{l}$ ou $a=A_{o}+\Delta$, onde $f^{l}($.$) é a função de$ transição inversa. Para os restantes intervalos de amplitude, $\left[A_{i}, A_{i}+\Delta[\right.$, a probabilidade é dada por:

$$
P_{i}(a)=\frac{2}{4 \cdot T}\left(f^{-1}(a)-f^{-1}(a-\Delta)\right)
$$

Considerou-se, ainda, que o tempo de subida é igual ao tempo de descida, e, consequentemente, contribuem similarmente para as probabilidades.

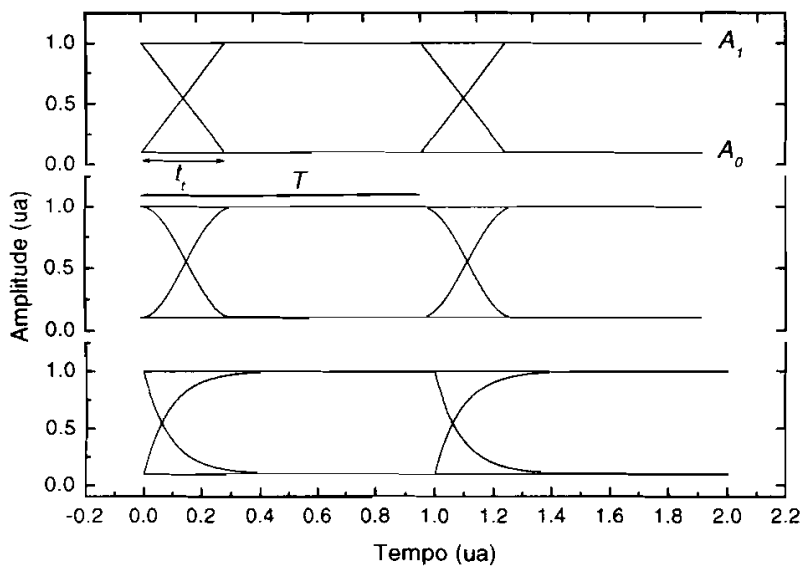

Figura 4. Diagramas de olho para os impulsos estudados: transição linear ideal (cima), transição coseno elevado (meio) e transição passa baixo (baixo).

As funções de transição consideradas e representadas na figura 4 são as seguintes:

$$
f(t)=A_{0}+\frac{A_{1}-A_{0}}{\tau \cdot T} \cdot t
$$

$$
\begin{gathered}
f(t)=\frac{A_{0}+A_{1}}{2}+\frac{A_{1}-A_{0}}{2} \cdot \sin \left(\frac{\tau \cdot \pi \cdot t}{T}-\frac{\pi}{2}\right) \\
f(t)=A_{0}+\left(A_{1}-A_{0}\right) \cdot \exp \left(-\frac{t \cdot \ln (1 / 9)}{\tau \cdot T}\right)
\end{gathered}
$$

para respectivamente as transições lineares, coseno elevado e passa baixo. As funções inversas das expressões (10), (11) e (12) podem ser facilmente obtidas analiticamente.

Após a aquisição do histograma de amplitude assíncrono sem ruído, a função do ruído é adicionada através da convolução do histograma sem ruído com o histograma da distribuição gaussiana do ruído. Aplicando o procedimento atrás descrito, foram simulados os histogramas assíncronos obtidos para impulsos com formato coseno elevado. $\mathrm{Na}$ figura 5 mostram-se os diagramas de olho síncronos determinados por simulação numérica e considerando uma situação ideal sem ruído dos impulso tipo coseno elevado. Nessa figura, são mostrados os diagramas correspondentes a diversos valores do parâmetro de transição.

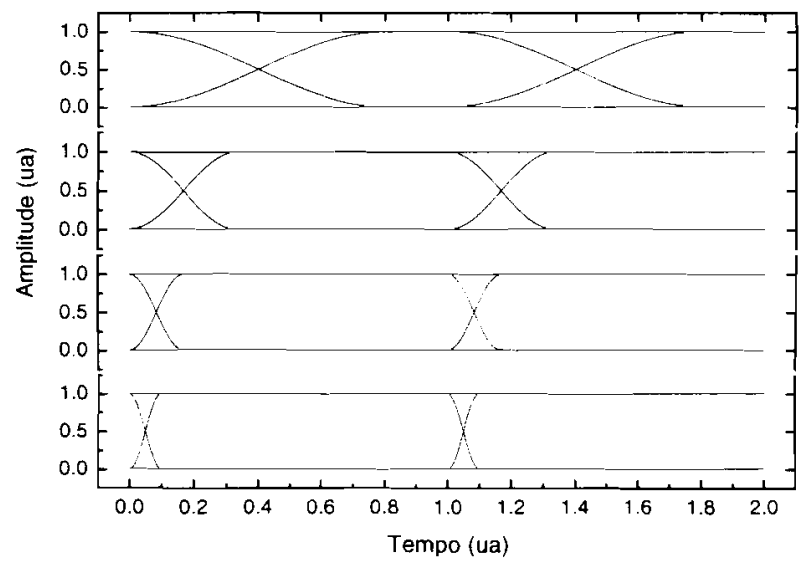

Figura 5. Diagramas de olho simulados para impulsos tipo coseno elevado, considerando uma situação ideal sem ruído. Os tempos de subida ( $10 \%$ a $90 \%$ ) são de $47 \%, 20 \%, 10$ $\%$ e $6 \%$ do período do bit, respectivamente, de cima para baixo.

Na figura 6 é mostrada a evolução dos histogramas assíncronos simulados, correspondentes a alguns diagramas de olho da figura 5 mas considerando a existência de ruído.

Nesses histogramas o factor $Q$ é de 5. As letras $p$ e $v$ assinalam respectivamente um pico e o vale do histograma. O mesmo procedimento foi aplicado para os impulsos com formatos do tipo passa baixo e tipo linear. Na figura 7 encontram-se resumidos os valores da razão entre a amplitude dos picos do histogramas e do vale, para os três tipos de impulsos considerados anteriormente e em função do tempo de subida dos impulsos.

A partir destes resultados, algumas características podem ser inferidas. A comparação destas curvas permite-nos verificar que a relação entre a amplitude dos picos e do vale dos histogramas difere para os impulsos tipo passa baixo e tipo coseno elevado. De referir que os impulsos do tipo linear são transições ideais sem aplicabilidade prática, sendo aqui referidas apenas como exemplo ilustrativo. Assim, nos impulsos do tipo passa baixo, a razão entre a amplitude do 


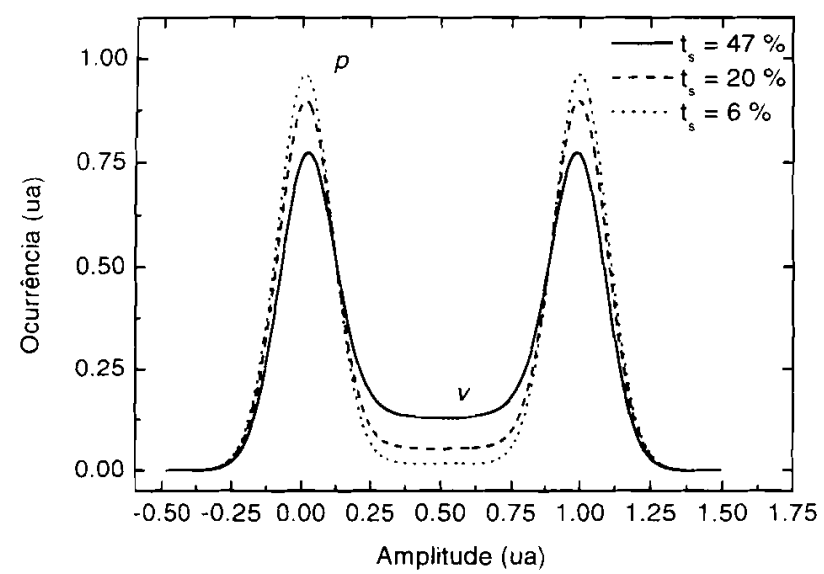

Figura 6. Histogramas assíncronos obtidos para diversos tempos de subida dos impulsos tipo coseno elevado e considerando um factor $Q$ de 5 .

pico e do vale rapidamente converge para um valor constante a partir de tempos de subida $(10 \% \rightarrow 90 \%)$ superiores a $25 \%$ do período do bit. Usualmente, os tempos de subida dos impulsos obtidos à saída dos geradores de sinal encontram-se nesta situação. Já no caso dos impulsos tipo coseno elevado, a convergência é mais lenta e como tal varia com os valores do período do bit.

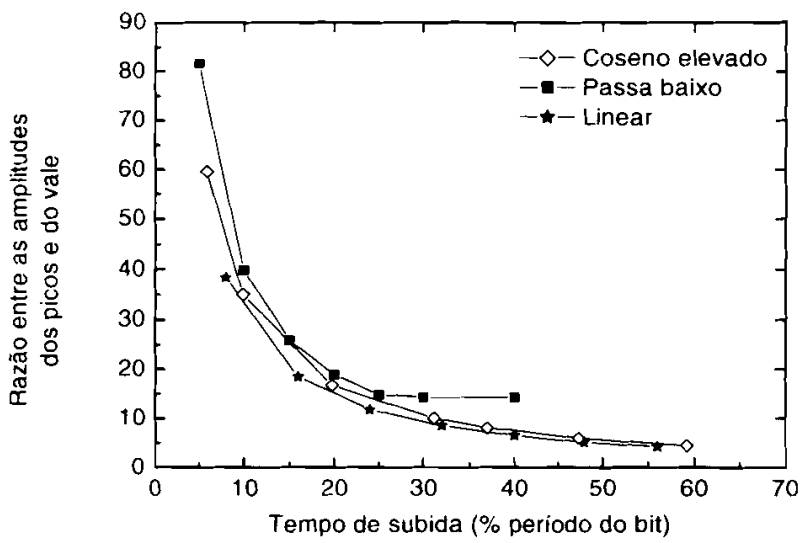

Figura 7. Razão entre a amplitude dos picos e do vale dos histogramas assíncronos em função do tempo de subida dos impulsos. As linhas são guias visuais.

Verifica-se que o ruído, também, contribui para a variação da razão entre a amplitude dos picos e do vale do histograma. Este modelo foi validado, através da sua verifi-cação experimental, onde foi calculado o factor $Q$ de um sinal a partir do seu histograma assíncrono. Assim, foram adquiridos os histogramas assíncronos de impulsos, cujas transições são do tipo passa baixo e coseno elevado.

Nas figuras 8 a) e 8 b) encontram-se respectivamente o diagrama de olho síncrono e assíncrono obtidos para os impulso do tipo coseno elevado. Estes impulsos apresentam um factor $Q$ de 5.16, medido para o caso síncrono através do osciloscópio com uma janela de integração de 40 ps. $\mathrm{O}$ tempo médio de subida ( $10 \% \rightarrow 90 \%$ ) dos impulsos é de $21.3 \mathrm{ps}$. Nas figuras $8 \mathrm{c}$ ) e $8 \mathrm{~d}$ ) mostram-se, respectivamente, o diagrama de olho síncrono e assíncrono obtidos para os impulso do tipo passa baixo. Estes impulsos apresentam um factor $Q$ de 5.23, medido para o caso síncrono através do osciloscópio com uma janela de integração de 40 ps. O tempo médio de subida $(10 \% \rightarrow 90 \%)$ dos impulsos é de 150 ps.

Através do processo de ajuste, os histogramas obtidos experimentalmente são simulados e os erros quadráticos entre os resultados experimentais e simulados são minimizados através de um algoritmo Nelder-Mead Simplex. No caso dos impulsos coseno elevado tal ocorre quando o tempo de subida simulado e o factor $Q$ simulado são de respectivamente 218 ps é 5.03 . No caso dos impulsos do tipo passa baixo a minimização ocorre quando o tempo de subida simulado e o factor $Q$ simulado são de respectivamente 144 ps é 5.28 .

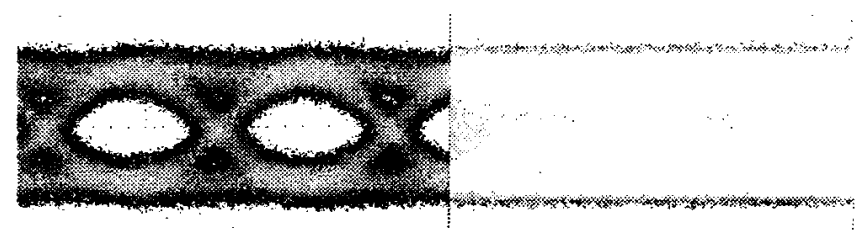

a)

b)

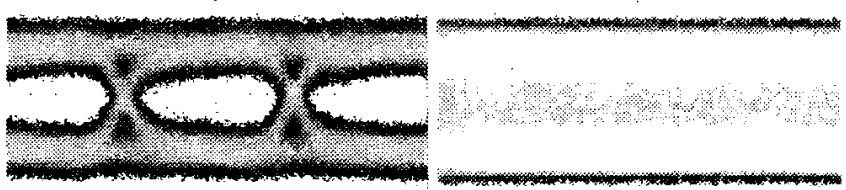

c)

d)

Figura 8. Diagramas de olho de impulsos tipo coseno elevado: a) síncrono e b) assíncrono. Diagramas de olho de impulsos tipo passa baixo: c) síncrono e d) assíncrono. Nos diagramas assíncronos são visíveis as janelas de integração e os histogramas obtidos.

A concordância entre os valores simulados e os valores experimentais pode ser observada na figura 9 , onde se mostra a sobreposição dos histogramas experimentais com o respectivo ajuste.

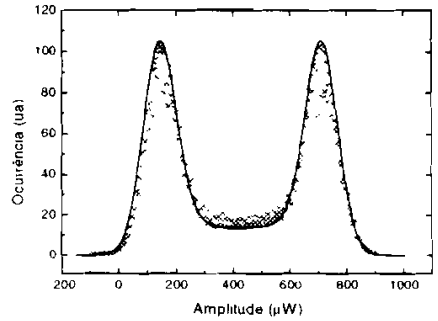

a)

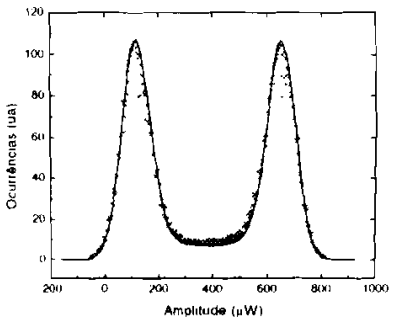

b)
Figura 9. Histogramas assíncronos experimentais e simulados, para impulsos do tipo: a) coseno elevado e b) passa baixo. Os pontos são dados experimentais e as linhas são os resultados do ajuste.

Estes resultados mostram que este método pode caracterizar com rigor as principais características dos impulsos, apresentando a vantagem de ser transparente ao formato do sinal. 


\subsection{FACTOR Q ASSÍNCRONO NA PRESEN- ÇA DE DEGRADAÇÃO DA FORMA DO IMPULSO}

Pretende-se, nesta secção, verificar a relação entre o factor $Q$ assíncrono e o factor $Q$ síncrono em sistemas de comunicações ópticas, onde exista uma degradação da forma dos impulsos, provocada pela dispersão cromática e efeitos não lineares. Para se analisar a relação entre o factor $Q$ síncrono e assíncrono, implementou-se o sistema de transmissão com $70 \mathrm{~km}$ que se encontra na figura 10 .

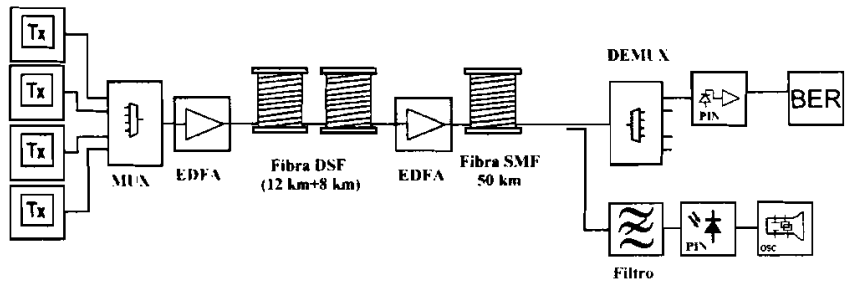

Figura 10. Esquema do sistema experimental implementado.

Os sinais ópticos de quatro lasers a emitirem respectivamente com os comprimentos de onda de $1547.72 \mathrm{~nm}$, $1549.32 \mathrm{~nm}, 1550.92 \mathrm{~nm}$ e $1552.52 \mathrm{~nm}$, foram modulados externamente com sequências PRBS de comprimento $2^{15}-1$ e com um ritmo de transmissão de $2.48832 \mathrm{Gbit} / \mathrm{s}$, ou seja STM-16. Os quatro sinais ópticos foram multiplexados e opticamente amplificados, antes de serem injectados em $20 \mathrm{~km}(12 \mathrm{~km}+8 \mathrm{~km})$ de fibra com dispersão deslocada (DSF). Posteriormente o sinal foi de novo opticamente amplificado e injectado em mais $50 \mathrm{~km}$ de fibra com dispersão padrão (SMF). Finalmente, o sinal foi desmultiplexado, detectado e a sua BER analisada. À entrada do DEMUX foi utilizado um acoplador de potência óptica de $10 \%$ que alimenta o monitor do desempenho.

O monitor de desempenho é constituído por um filtro óptico sintonizável com um isolamento entre canais adjacentes superior a $46 \mathrm{~dB}$ e que permite sintonizar o canal a analisar, um fotodíodo com uma largura de banda superior a $15 \mathrm{GHz}$ e um osciloscópio digital com uma largura de banda de $40 \mathrm{GHz}$ a funcionar num modo de varrimento livre com uma taxa de amostragem de $500 \mathrm{KHz}$.

Os testes foram realizados em três situações diferentes de propagação: ligação directa, propagação não linear e propagação linear. Na situação de ligação directa o monitor de desempenho é colocado logo após o MUX. No segundo caso, foi injectada uma elevada potência óptica na fibra DSF ( $5 \mathrm{dBm}$ por canal). Esta potência é suficiente para gerar produtos de mistura de quatro ondas na fibra DSF e. também, criar auto modulação de fase, bem como modulação cruzada de fase, ao longo da propagação na fibra SMF. Na terceira situação, foi injectada na fibra DSF uma potência óptica de $-10 \mathrm{dBm}$ por canal, sendo este valor considerado suficientemente baixo para permitir propagação linear. $\mathrm{O}$ amplificador óptico intermédio funciona num regime de saturação com uma potência óptica de saída de 13 $\mathrm{dBm}$.

Na figura 11 mostram-se os espectros ópticos nas três situações de propagação atrás referidas. $\mathrm{O}$ sinal eléctrico detectado no fotodíodo do monitor de desempenho foi amostrado assincronamente e caracterizado por amostras obtidas em instantes arbitrários. É de referir que na construção do histograma de amplitude a janela de amostragem deve ser uma fracção da duração do período das anmutras para evitar a perda de informação devido à acumulação de amostras. A sub-amostragem não degrada o desempenho deste método mas uma precisão elevada exige um número elevado de amostras para se obter toda a estatística do sinal (neste caso, foram utilizadas 1 milhão de amostras).

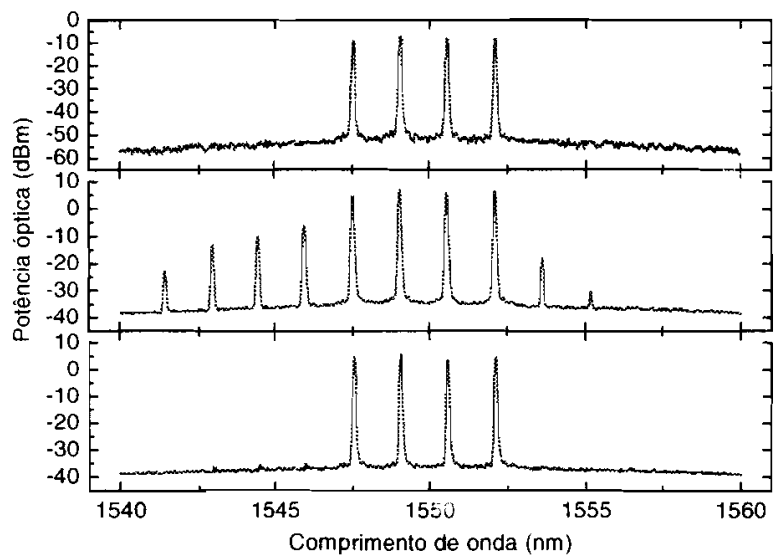

Figura 11. Espectros ópticos à saída do multiplexador (topo), no monitor de desempenho para o regime de propagação não linear (meio) e para o regime de propagação linear (base).

Foi, seguidamente, construído um histograma de amplitude com um milhão de amostras, utilizando para o efeito uma janela de integração com 40 ps. O histograma normalizado assim obtido foi ajustado a duas funções gaussianas. $\mathrm{O}$ ajuste foi realizado considerando todos os pontos com um valor superior a 0.2 e permitiu obter os valores médios e os desvios padrão dos dois níveis lógicos. O factor $Q_{\text {Asin }}$ fol, seguidamente. calculado através da expressão (6). O mesmo canal é sincronamente detectados e a taxa de erro do bit medida com um analisador de BER. O factor $Q_{\sin }$ foi calculado assumido uma aproximação gaussiana para o ruído, utilizando a função inversa da expressão (7) [7].

As figuras 12 a) e 12 b) mostram respectivamente os diagramas de olho assíncrono e síncrono, para o canal com o comprimento de onda de $1547.72 \mathrm{~nm}$, no regime de propagação não linear, após propagação em $70 \mathrm{~km}$ de fibra. $\mathrm{O}$ factor $Q$ foi, assim, determinado para diversos valores da potência óptica incidente no receptor (e no monitor de desempenho). $O$ mesmo procedimento foi realizado para o regime de propagação linear e para a situação de ligação directa. $O$ factor $Q$ assíncrono determinado reflecte a degradação do sinal provocada pela acumulação de ruído e distorção da forma dos impulsos, relacionada com a dispersão cromática e efeitos não lineares.

$\mathrm{Na}$ figura 13 encontra-se a relação entre o factor $Q$ assíncrono medido no monitor de desempenho e o factor $Q$ síncrono calculado a partir da BER. $O$ declive das rectas ajustadas aos vários pontos é o mesmo para os três regimes de propagação (1.225) e o coeficiente de correlação é superior a 0.9999 . Observa-se que o factor $Q$ assíncrono decresce linearmente com o factor $Q$ síncrono como resultado da degradação da relação sinal / ruído, para as três 


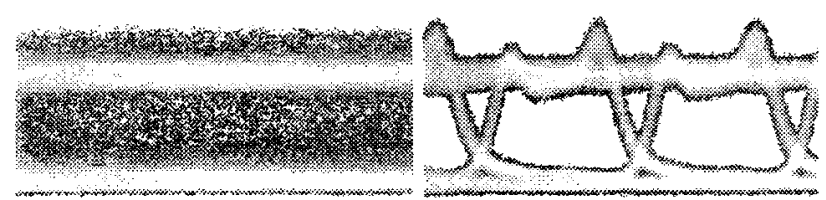

a)

b)

Figura 12. Diagramas de olho para o regime não linear: a) amostragem assíncrona no monitor de desempenho e b) amostragem síncrona no receptor. Os histogramas de amplitude encontram-se sobrepostos no lado esquerdo das imagens.

situações de propagação. Também se observa que para um determinado valor do factor $Q$ síncrono, o valor do factor $Q$ assíncrono diminui à medida que a degradação do sinal aumenta.

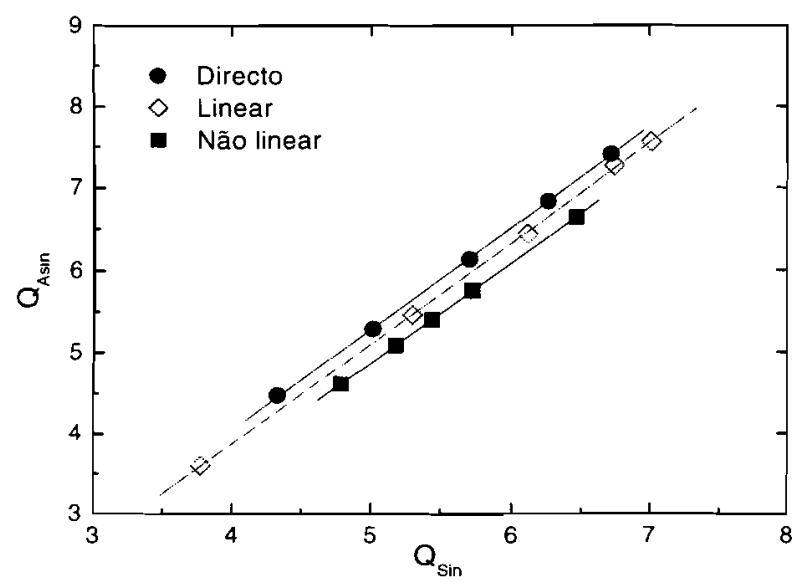

Figura 13. Relação entre o factor $Q$ assíncrono e síncrono. Os pontos são dados experimentais e as rectas o respectivo ajuste linear.

Assim, pode-se afirmar que o factor $Q$ assíncrono reflecte a degradação da BER causada pelos efeitos da dispersão cromática, ruído e não linearidades da fibra. Tal indica que os histogramas de amplitude assíncronos reflectem distorções da forma de onda dos impulsos e. consequentemente, podem ser utilizados para monitorização óptica do desempenho [14].

\subsection{ESTIMATIVA DA BER}

Como foi reportado anteriormente, os histogramas de amplitude assincronamente amostrados reflectem a degradação do desempenho dos sinais ópticos. Pretende-se, agora, obter directamente a BER do canal óptico [15]. Assim, após a detecção, o sinal eléctrico é assincronamente amostrado e o histograma de amplitude é construído, como descrito na subsecção anterior. A parte inferior do histograma, correspondente aos pontos com menos de $20 \%$ da sua amplitude máxima, é removida para eliminar os pontos de cruzamento correspondentes às transições entre os dois níveis lógicos. A função densidade de probabilidade é, então. calculada a partir dos restantes pontos [10]. Nesta fase, os valores médios e os desvios padrão para ambos os símbolos lógicos poderiam ser calculados a partir da função densidade de probabilidade e o factor $Q$, bem como o nível óptimo de decisão obtido, como na secção anterior. No entanto, na presença de distorção da forma dos impulsos ou interferência entre símbolos, o factor $Q$ e a BER não têm uma relação directa, analítica e exacta entre si. Como estes efeitos alargam a variância estimada, os parâmetros $\mu_{l}$ e $\mu_{0}$ devem ser tratados como variáveis aleatórias, em vez de valores constantes [16-18]

Os valores discretos das funções densidade de probabilidade representam os valores amostrados de um sinal distorcido, com $m$ amostras no símbolo lógico "1" e $n$ amostras no símbolo lógico " 0 ". Cada amostra tem associado um ruído com distribuição gaussiana e com variância para o símbolo " 0 " e "l" respectivamente de $\sigma_{(0, n)}{ }^{2}$ e $\sigma_{(l, m)}{ }^{2}$, dadas por [19]:

$$
\begin{array}{r}
\sigma_{(0, n),(1, m)}^{2}=2 \cdot S \cdot I_{(0, n),(1, m)} \cdot p_{r} \cdot B_{e}+ \\
S^{2} \cdot p_{e}^{2} \cdot B_{e}\left[B_{o}-\left(\frac{B_{e}}{2}\right)\right]
\end{array}
$$

Estas variâncias dependem da largura de banda dos filtros eléctricos e ópticos (respectivamente $B_{\rho}$ e $B_{o}$ ), dos valores amostrados da corrente eléctrica $\left(I_{0 . I}\right)$, da responsividade do fotodíodo $(S)$ e da densidade espectral de potência do ruído resultante da emissão espontânea dos amplificadores ópticos $\left(p_{r}\right)$. Este modelo para a variância considera os batimentos da ASE com a ASE a frequências diferentes e do sinal com a ASE. Assim, na presença de distorções a função densidade de probabilidade do sinal é gaussiana, sendo dada pela convolução de $N$ funções densidade de probabilidade, também, do tipo gaussiano. A partir destas novas funções densidade de probabilidade, é possível estimar em função do nível de decisão $I_{d}$, a BER utilizando a seguinte expressão [19]:

$$
\begin{aligned}
\operatorname{BER}\left(I_{d}\right)= & \frac{1}{2} \cdot \sum_{n} H\left(I_{0, n}\right) \operatorname{erfc}\left(\frac{I_{d}-I_{0, n}}{\sqrt{2} \cdot \sigma_{0, n}}\right)+ \\
& \frac{1}{2} \cdot \sum_{m} H\left(I_{1, m}\right) \operatorname{erfc}\left(\frac{I_{d}-I_{1, m}}{\sqrt{2} \cdot \sigma_{1, m}}\right)
\end{aligned}
$$

onde, $N=m+n$, é o número total de valores discretos. As ocorrências relativas normalizadas das amostras de corrente eléctrica satisfazem a relação:

$$
\sum_{n} H\left(I_{0, n}\right)=\sum_{m} H\left(I_{1 . m}\right)=\frac{1}{2}
$$

Como só existem pontos experimentais próximos dos níveis lógicos dos símbolos " 0 " e " 1 " é necessário utilizar um processo de extrapolação das caudas das funções densidade de probabilidade e extrapolar a BER mínima correspondente ao nível de decisão óptimo [20]. A aproximação atrás descrita foi aplicada aos dados do histo-grama e a BER estimada. A BER foi, também, medida para o mesmo canal no receptor óptico. Este procedimento foi realizado para vários valores da potência óptica incidente no receptor e para os regimes de propagação não linear, linear e directo. Na figura 14 os resultados estimados para a BER são comparados com os valores experimentais para os três regimes de propagação.

Em todas as condições de propagação, o erro relativo máximo entre os dados experimentais e os pontos estimados 
é inferior a $\pm 5 \%$. Neste último gráfico é visível um ponto que cai fora do intervalo de erro relativo, e que resulta de erros experimentais provavelmente associados à aquisição do diagrama de olho assíncrono. Assim, a partir da monitorização do canal óptico e conhecendo as características do receptor tais como o filtro óptico, o fotodetector e o filtro eléctrico é possível determinar de forma analítica a taxa de bits errados, o que demonstra a capacidade de estimar a BER a partir de histogramas de amplitude assíncronos [21].

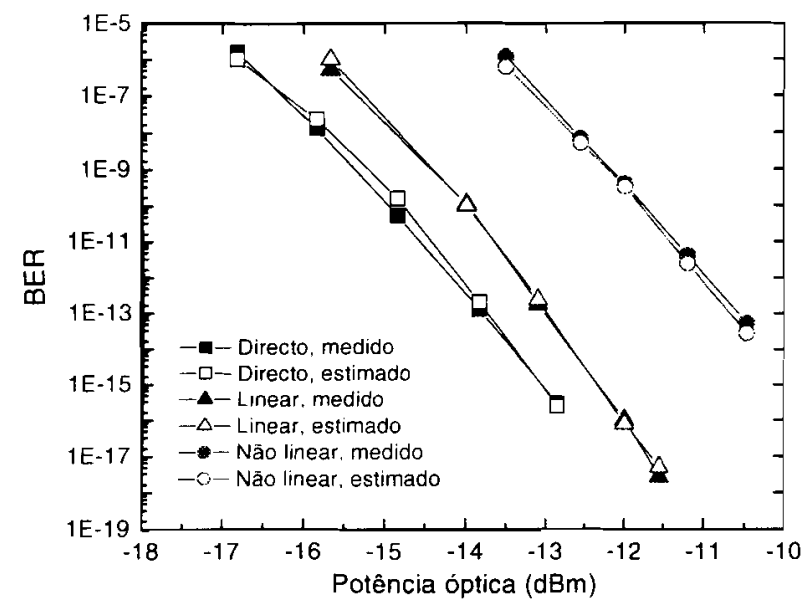

Figura 14. BER medida e estimada em função da potência óptica injectada no receptor, para os três regimes de propagação. As linhas são guias visuais.

\section{CONCLUSÃo}

As funções de monitorização do desempenho encontramse numa fase de migração do domínio eléctrico para o domínio óptico. Neste trabalho, demonstrou-se o conceito de monitorização óptica do desempenho, baseada na análise de histogramas de amplitude assincronamente amostrados. O modelo utilizado reflecte a degradação do sinal relacionada com a acumulação de ruído e distorções da forma de onda como resultado da dispersão cromática e efeitos não lineares. Os resultados obtidos são promissores e indicam que esta técnica pode ser empregue para monitorização óptica do desempenho nas futuras rede ópticas transparentes de alta velocidade

\section{AGRADECIMENTOS:}

Os autores agradecem o apoio financeiro providenciado pela FCT a níveli individual (PRAXIS XXI/BD/17227/98) através dos projectos WIDCOM (POSI/35574/ 99/CPS/ 2000) e WO-Net (POSI/2001/CPS/40009) a colaboração do Instituto de Telecomunicações - Aveiro, da Universidade de Aveiro e da Portugal Telecom Inovação.

\section{REFERÊNCIAS}

[1] Dietrich Marcuse, "Derivation of Analytical Expressions for the Bit-Error Probability in Lightwave Systems with Optical Amplifiers", OSA/IEEE Journal of Lightwave Technology, vol.8, pp.1816-1823, December 1990.

[2] A. Bononi, Optical Networking, Springer-Verlag, 1999.
[3] W. D. Cornwell, I. "Andonovic, Interferometric noise for a single interfer: Comparison between theory and experiment". IEE Electronics Letters, vol.32, pp.1501-1502, April 1996.

[4] Nam Su Moom, Takahide Sakamoto, Yuichi Takashima, Kazuro Kikuchi, "Experimental Verification of Gaussian Approximation Model of Multiple Intraband Crosstalk in Wavelength-Division Multiplexed Networks Using Recirculating Fiber Loop", IEEE Photonics Technology Letters. vol.13, pp.1038-1040, September 2001

[5] G. Jacobsen, L. Gillner. O. Fransson, "On the Use of OSNR for BER Monitoring in WDM Systems an Optical Networks", Journal of Optical Communications, vol.21, pp.20-23. January 2000.

[6] Shoko Ohteru, Noboru Takachio, "Optical Signal Quality Monitoring Using Direct Q-Factor Measurement". IEEE Photonics Technology Letters, vol.11, pp.1307-1309. October 1999.

[7] F. Matera, M. Settembre, "Role of Q-Factor and of Time Jitter in the Performance Evaluation of Optically Amplified Transmission Systems", IEEE Journal of Selected Topics in Quantum Electronics, vol.6, pp.308-316, February 2000.

[8] Seiji Norimatsu. Masanori Maruoka, "Accurate Q factor Estimation of Optically Amplified Systems in the Presence of Waveform Distortion", OSA/IEEE Journal of Lightwave Technology, vol.20, pp.19-27, January 2002

[9] N. Hanik, A. Gladisch, C. Caspar, B. Strebe], "Applications of Amplitude Histograms to Monitor Performance of Optical Channels", IEE Electronic Letters, vol.35, pp. 403-404, February 1999.

[10] I. Shake, H. Takara, S. Kawanishi. Y. Yamabayashi, "Optical Signal Quality Monitoring Method Based on Optical Sampling", IEE Electronics Letters, vol.34, pp.2152-2153, June 1998.

[11] Ippei Shake, Hidehiko Takara. Kentaro Uchiyama. Yoshiaki Yamabayashi, "Quality Monitoring of Optical Signals Influenced by Chromatic Dispersion in a Transmission Fiber Using Averaged Q-Factor Evaluation", IEEE Photonics Technology Letters, vol.13, pp.385-387. March 2001.

[12] Richard Habel, Kim Roberts, Alan Solheim, James Harley, "Optical Domain Performance Monitoring", OFC 2000. WK3-1, pp.174-175, Baltimore, USA, March 2000.

[13] António Teixeira, Paulo André, Mário Lima, José da Rocha, J. Pinto, "Characterization of High Bit Rate Optical Signals by Low Rate Asynchronous Sampling", LEOS 2002, Glasgow, United Kingdom, November 2002.

[14] P. S. André, A.L.J. Teixeira, M.J.N. Lima, J.L. Pinto, J.R.F. da Rocha, "Optical Performance Monitor Based on Asynchronous Detection", 14 th IEEE LEOS Annual Meeting, pp. 30-31, San Diego, USA, November 2001.

[15] P. S. André and J. L. Pinto, A. L. J. Teixeira, M. J. N. Lima and J. F. da Rocha, "Bit error rate assessment in DWDM transparent networks using optical performance monitor based in asynchronous sampling", $O F C$ 2002, ThGG95, pp.749-750, Anaheim, USA, March 2002

[16] T. Takahashi, M. Aoyama, M. Murakami, M. Ameniya "Modelling of intersymbol interference effect on signal to noise ratio measurement in long haul optical amplifier systems", IEE Electronics Letters, vol.31, pp.2195-2197, July 1995.

[17] C. J. Anderson, J. A. Lyle, "Technique for evaluating system performance using $Q$ in numerical simulations exhibiting intersymbol interference", IEE Electronics Letters, vol.30, pp.71-72, January 1994.

[18] E. G. Shapiro, M. P. Fedoruk, S. K. Turitsyn, "Numerical estimate of BER in optical systems with strong patterning effects", IEE Electronics Letters, vol. 37, pp.1179-1180, March 2001

[19] M. Rasztovits-Weich, K. Studer, W. R. Leeb, "Bit error probability estimation algorithm for signal supervision in all- 
optical network"., IEE Electronics Letters, vol.35, pp. 1754 1755. May 1999.

[20] Neal S. Bergano. F. W. Kerfoot, C. R. Davidson, "Margin Measurements in Optical Amplifiers Systems", IEEE Photonics Technology Letters, vol.5, pp.304-306, March 1993.

[2I] P. S. André, A. L. J. Teixeira, Teresa Almeida, M. Pousa. J. F. da Rocha and J. L. Pinto, "Optical signal quality monitor for transparent DWDM networks based on asynchronous sampled histograms", OSA Journal of Optical Networks, vol.1, pp.118-128. March 2002.

Paulo André licenciou-se em Engenharia Física em 1996 e Doutorou-se em Física em 2002 na Universidade de Aveiro. É Investigador Auxiliar no Instituto de Telecomunicações de Aveiro e Professor Auxiliar Convidado a tempo parcial na Universidade de Aveiro.

António Teixeira licenciou-se em Engenharia Eletrónica e Telecomunicações em 1994 e Doutorou-se em Eletrónica e Telecomunicações em 1999 na Universidade de Aveiro. É Professor Auxiliar na Universidade de Aveiro.
Mario Lima licenciou-se em Engenharia Eletrónica e Telecomunicações em 1994 e finalizou o Mestrado em Eletrónica e Telecomunicações em 1998 na Universidade de Aveiro. É Assistente Convidado na Universidade de Aveiro.

José Ferreira da Rocha finalizou o Mestrado em Sistemas de Telecomunicaçōes em 1980 e o Doutoramento em Engenharia Eléctrica em 1983 na Universidade de Essex, Inglaterra. É Professor Catedrático na Universidade de Aveiro.

João Lemos Pinto licenciou-se em Engenharia Eletrotécnica em 1975 na Universidade do Porto e Doutorou-se em Física Aplicada em 1987 na Universidade de Hull, Inglaterra. É Professor Catedrático na Universidade de Aveiro.

Teresa Almeida licenciou-se em Física Aplicada - Óptica e eletrónica em 1986 na Universidade do Porto. É Investigadora na Portugal Telecom Inovação desde 1989.

Marcelino Pousa licenciou-se em Física Aplicada - Óptica e eletrónica em 1984 na Universidade do Porto. É Investigador na Portugal Telecom Inovação desde 1986. 